\title{
Moduladores CFTR (cystic fibrosis transmembrane conductance regulator): presente y futuro en la terapia de fibrosis quística. Revisión
} Cystic fibrosis transmembrane conductance regulator modulators: Present and future in cystic fibrosis treatment. A review

\section{Dra.Diana Dela Hoz ${ }^{a}$, Dra. Milena VillamilOsorio ${ }^{a, b}$ y Dra. SoniaM. Restrepo-Gualteros ${ }^{a, b}$}

\section{RESUMEN}

Los moduladores cystic fibrosis transmembrane conductance regulator (CFTR) representan el presente y el futuro del manejo farmacológico para los pacientes con fibrosis quística. El objetivo de esta publicación es realizar una revisión de esta opción terapéutica. Se revisaron artículos científicos consultando las bases de datos MedLine, información disponible a través de la página oficial Cystic Fibrosis Foundation, desde 2009 hasta 2018, en el idioma inglés. Sin restricciones respecto al tipo de estudio, se seleccionaron 12 artículos que incluyeron información sobre el estado actual de la investigación sobre moduladores CFTR. Actualmente, están aprobados por la Food and Drug Administration tres moduladores: ivacaftor, lumacaftor + ivacaftor y tezacaftor + ivacaftor, y hay otros 11 en diferentes fases de estudio. La terapia con moduladores CFTR es una realidad en desarrollo que apunta al máximo objetivo de la medicina personalizada y que promete mejorar la calidad de vida de pacientes con fibrosis quística.

Palabras clave: fibrosis quistica, mutación, terapia, regulador de conductancia transmembrana de fibrosis quística.

a. Universidad

Nacional de Colombia,

Departamento de Pediatría, Bogotá DC, Colombia.

b. Fundación Hospital Pediátrico de la Misericordia, Bogotá DC, Colombia.

Correspondencia: Dra. Diana De la Hoz: dcdeg@unal.edu.co

Financiamiento: Ninguno.

Conflicto de intereses: Ninguno que declarar.

Recibido: 20-3-2018 Aceptado: 20-10-2018 http:/ / dx.doi.org/10.5546/aap.2019.e131

Texto completo en inglés:

http:/ / dx.doi.org/10.5546/ aap.2019.eng.e131

Cómo citar: De la Hoz D, Villamil Osorio M, RestrepoGualteros S1. Moduladores CFTR (cystic fibrosis transmembrane conductance regulator): presente y futuro en la terapia de fibrosis quística. Revisión. Arch Argent Pediatr 2019;117(2):e131-e136.

\section{INTRODUCCIÓN}

La supervivencia de los pacientes con fibrosis quística (FQ) ha mejorado en los últimos 30 años. En esta enfermedad, existe un defecto del gen que codifica para la proteína cystic fibrosis transmembrane conductance regulator (CFTR), que cumple la función de servir como canal transmembrana de iones cloro. Cada día, se conoce más del funcionamiento de esta proteína, lo que ha permitido dirigir las herramientas terapéuticas en función de su restauración. ${ }^{1,2}$

Los moduladores de CFTR se han encontrado como el presente y el futuro de la terapia farmacológica para los pacientes con FQ. En 2012, se dio la aprobación por parte de la Food and Drug Administration (FDA) del ivacaftor, $y$, desde entonces, se dispone de un mayor número de fármacos que se encuentran en estudio y que apuntan a la corrección del defecto proteico en diferentes puntos.

\section{PROTEÍNA cystic fibrosis} transmembrane conductance regulator

La proteína CFTR es una glicoproteína de 1480 aminoácidos, compuesta de 5 dominios: dos dominios transmembrana, dos sitios de unión de nucleótidos y un dominio regulador. ${ }^{2}$

Hasta el momento, se han descrito más de 1700 mutaciones causantes de FQ, agrupadas en clases:

- Mutación clase I: Es un defecto cuantitativo, que no permite una adecuada longitud de la proteína, y resulta, por lo tanto, en una proteína truncada; el mecanismo es la formación de codones de detención. De estas, la más común es la mutación G542X.2-4

- Mutación clase II: Es un defecto cuantitativo, con producción de una proteína con mal plegamiento, que no alcanza la superficie al 
sufrir degradación intracelular. La mutación F508 es la más representativa de esta clase..$^{2-4}$

- Mutación clase III: Es un defecto cualitativo. No hay respuesta a la estimulación por la monofosfato adenosín ciclasa. La mutación G551D es la más común de este tipo. ${ }^{2-4}$

- Mutación clase IV: Defecto cualitativo con una proteína correctamente localizada, pero con un error en la conductancia. R117H es la más común..$^{2-4}$

- Mutación clase V: Se muestra con una cantidad reducida de CFTR en la membrana. ${ }^{2-4}$

- Mutación clase VI: Aumento del recambio de CFTR funcionales en la membrana. ${ }^{2-4}$

\section{MODULADORES cystic fibrosis transmembrane conductance regulator}

Desde hace 10 años, ha cambiado significativamente la terapéutica de $\mathrm{FQ}$, con el surgimiento de moduladores CFTR, basados en la función de la proteína alterada según los 6 tipos de mutaciones descritos, que se consideran el ideal de la medicina personalizada, según la cual la utilización del fármaco depende de la genotipificación del defecto (Tabla 1). ${ }^{1-4}$

\section{AGENTES Read Through}

Las mutaciones de parada prematura o nonsense constituyen el 5-10\% de todas las mutaciones de CFTR y se enmarcan dentro de la clase I. Los medicamentos conocidos como agentes Read Through actúan como agentes de la lectura a través del ribosoma y suprimen los codones de parada prematura, lo que permite obtener una proteína de longitud normal que pueda ser transportada a la superficie. ${ }^{2,3}$

Dentro de este grupo, se estudió, inicialmente, el efecto de aminoglucósidos en bajas dosis (la gentamicina cuenta con mayor evidencia) y se demostró una reducción significativa de la diferencia del potencial nasal. Sin embargo, se considera que los beneficios clínicos se ven limitados por la necesidad de aplicación intravascular o intramuscular, como también por la falta de potencia y toxicidad renal. ${ }^{2,3}$

Ataluren (PCT124) fue un medicamento diseñado, inicialmente, como agente Read Through, pero los resultados no fueron los esperados. En 2016, se consideró que la evidencia disponible no permitía determinar el efecto en los pacientes y, el 3 de marzo de 2017, se anunció que la compañía que trabajaba en su desarrollo lo suspendió, dado que, en el ensayo clínico fase 3, no mostró una mejoría significativa en la función pulmonar ni en la disminución de las exacerbaciones. Este ensayo se había iniciado en 2015 con pacientes con una mutación del grupo clase I..$^{5,6}$

Hasta la fecha, no hay medicamentos disponibles para la mutación clase I, la cual está presente en alrededor del $10 \%$ de los pacientes con FQ. ${ }^{5,6}$

\section{POTENCIADORES cystic fibrosis transmembrane conductance regulator}

El fármaco representativo de este grupo es el ivacaftor, identificado a través de un proceso de selección de alto rendimiento entre más de 228000 compuestos, con el que, posteriormente, se trabajó para conseguir un perfil farmacocinético adecuado. ${ }^{3,7}$

En células de epitelio bronquial humano con mutaciones G551D/F508del, ivacaftor ha demostrado un aumento de la superficie líquida a niveles equivalentes a, aproximadamente, la mitad del nivel observado en células no-fibrosis quística (no-FQ), como también un aumento de la frecuencia de movimiento ciliar hasta cerca de los valores normales. ${ }^{7}$

TABLA 1. Clases de mutaciones y ejemplo de terapia

\begin{tabular}{|c|c|c|c|c|c|c|}
\hline & Clase I & Clase II & Clase III & Clase IV & Clase V & Clase VI \\
\hline Tipo de mutación & $\begin{array}{l}\text { No se sintetiza } \\
\text { la proteína } \\
\text { Nonsense, } \\
\text { frameshift. }\end{array}$ & $\begin{array}{l}\text { Defecto de } \\
\text { plegamiento } \\
\text { Missense, } \\
\text { deleción. }\end{array}$ & $\begin{array}{l}\text { Defecto de } \\
\text { apertura } \\
\text { del canal } \\
\text { Missense, } \\
\text { cambio de } \\
\text { aminoácidos. }\end{array}$ & $\begin{array}{l}\text { Defecto de } \\
\text { transporte } \\
\text { de iones } \\
\text { Missense, } \\
\text { cambio de } \\
\text { aminoácidos. }\end{array}$ & $\begin{array}{l}\text { Descenso de } \\
\text { síntesis de } \\
\text { proteínas } \\
\text { Splicing. }\end{array}$ & $\begin{array}{l}\text { Descenso de } \\
\text { la vida media } \\
\text { de la proteína } \\
\text { Missense, } \\
\text { cambio de } \\
\text { aminoácidos. }\end{array}$ \\
\hline Terapia & $\begin{array}{l}\text { Terapia } \\
\text { génica }\end{array}$ & $\begin{array}{l}\text { Lumacaftor, } \\
\text { Ivacaftor, } \\
\text { Tezacaftor } \\
\text { Terapia génica }\end{array}$ & $\begin{array}{l}\text { Ivacaftor } \\
\text { Terapia } \\
\text { génica }\end{array}$ & $\begin{array}{l}\text { Ivacaftor } \\
\text { Terapia génica } \\
\text { Tezacaftor } \\
\text { (mutaciones } \\
\text { específicas) }\end{array}$ & $\begin{array}{l}\text { Terapia génica } \\
\text { Tezacaftor } \\
\text { mutaciones } \\
\text { específicas) }\end{array}$ & $\begin{array}{l}\text { Terapia } \\
\text { génica }\end{array}$ \\
\hline
\end{tabular}


Entre los estudios de ivacaftor, se resaltan dos, ambos doble ciego, controlados, aleatorizados, fase III, que son el ENVISION y el STRIVE. En ambos, se observó una reducción significativa del cloro en sudor. Así, también se documentaron aumentos absolutos de volumen espiratorio forzado previsto en el primer segundo (VEF1) del 10,4\% (STRIVE) y del 10,7 \% (ENVISION). Ivacaftor también produjo una reducción significativa del $55 \%$ en las exacerbaciones pulmonares en STRIVE. Un estudio posterior, el PERSIST, que incluyó a 144 adolescentes / adultos y a 48 niños que presentaban la mutación G551D y que, previamente, habían sido formulados con ivacaftor como parte de STRIVE y ENVISION, demostró una mejoría sostenida de la función pulmonar, el peso y las exacerbaciones pulmonares hasta las 144 semanas de tratamiento. ${ }^{3,4}$

Cabe notar que el efecto benéfico de ivacaftor no se limita a la mutación G551D, sino que se extiende a un número amplio de mutaciones, que incluyen G551S, G1349D, G1244E, G970R, G178R, S549N, S549R, S1251N y S1255P, entre otras. Este efecto potenciador abarca, a su vez, incluso mutaciones de otras clases, como es el caso de las mutaciones clase IV, R117H y D1152H, en las que ha demostrado una respuesta favorable. ${ }^{4}$

En mayo de 2017, la FDA aprobó el uso de este medicamento para 23 mutaciones más, en los que los estudios evidenciaron beneficio. Actualmente, está evaluando su uso en pacientes con 6 mutaciones más incluidas en el grupo $\mathrm{V}$ (para la totalidad de este grupo, aún no se ha desarrollado ningún medicamento específico). ${ }^{8}$

\section{CORRECTORES cystic fibrosis transmembrane conductance regulator - TERAPIA COMBINADA}

Estos medicamentos están diseñados para aumentar la cantidad de proteína "funcional" que alcanza la membrana apical celular. En este grupo, el objetivo son las mutaciones clase II, como F508del. ${ }^{2,3}$

El lumacaftor (VX-809) es una molécula, cuyos estudios iniciales in vitro evidenciaron una mejoría de la maduración del CFTR en 7,1 veces, que se acompañó de una mejoría en 5 veces del transporte de cloro. Parecería que lumacaftor actúa a nivel del primer dominio transmembrana (Membrane Spanning Domain; MSD, por sus siglas en inglés), lo que da lugar a una corrección parcial del defecto de plegamiento del CFTR, mediante la mejora de la interacción entre los dominios transmembrana MSD1 y MSD2 y el dominio de unión de nucleótidos NBD1 (nucleotide-binding domains). ${ }^{4}$ Este medicamento en monoterapia no demostró diferencias significativas en cuanto a los valores de la prueba del sudor, el VEF1, el cuestionario de síntomas pulmonares de FQ (Revised Cystic Fibrosis Quality of Life Questionnaire in Adolescents and Adult, CFQ-R) ni en la diferencia de potenciales nasales. ${ }^{9}$

Uno de los primeros estudios en demostrar la eficacia de la terapia combinada incluyó tres cohortes sucesivas; la cohorte 1 incluyó a 64 participantes homocigotos para la mutación F508del, y las cohortes 2 y 3 incluyeron, en total, a 124 pacientes tanto homocigotos como heterocigotos para la misma mutación. El tratamiento con lumacaftor + ivacaftor $(\mathrm{L}+\mathrm{I})$ disminuyó la concentración media de cloro en sudor en $9,1 \mathrm{mmol} / 1(\mathrm{p}<0,001)$ en la cohorte 1 ; en las cohortes 2 y 3 , la concentración de cloro en sudor no disminuyó significativamente. A su vez, en los pacientes homocigotos (en especial, de la cohorte 2), se observó una mejoría del VEF1 $(p=0,013)$, cambio que no se presentó en el grupo de pacientes heterocigotos para la misma mutación. Finalmente, se concluyó que la terapia combinada mejoraba el VEF1 de pacientes con FQ que eran homocigotos para F508del, con un efecto modesto sobre la concentración de cloro en sudor. ${ }^{10}$

Los resultados de los estudios TRAFFIC y TRANSPORT fueron publicados en 2015 y documentaron la seguridad y eficacia de la terapia combinada. Incluyeron a 1108 pacientes mayores de 12 años homocigotos para la mutación F508del. El punto final primario fue el cambio absoluto del VEF1 en la semana 24. En ambos estudios, hubo mejoras significativas en el punto final primario; la diferencia entre la terapia combinada y el placebo con respecto al cambio del VEF1 previsto varió de 2,6 a 4,0 puntos porcentuales ( $\mathrm{p}<0,001$ ). La tasa de exacerbaciones pulmonares fue del $30 \%$ al $39 \%$ más baja en los grupos de L + I que en el grupo de placebo. ${ }^{11}$

Un estudio observacional multicéntrico evaluó los efectos de la administración de la combinación de L + I, midiendo la cantidad de eventos adversos, la interrupción del tratamiento, el VEF1 y el índice de masa corporal (IMC) un mes y tres meses después del inicio de lumacaftor + ivacaftor, en adultos con FQ y VEF1 por debajo del $40 \%$ del pronosticado. El estudio demostró que los pacientes que utilizaron la combinación L + I presentaron eventos adversos en el $51 \%$ de los casos, que causaron que, por 
lo menos, el $24 \%$ suspendiera el tratamiento. No obstante, quienes continuaron el manejo con lumacaftor + ivacaftor presentaron una mejoría significativa del VEF1 (+ el 3,19\% a los 3 meses), que fue comparable a lo observado en pacientes reclutados en estudios clínicos de fase 3 y que podría tener muy buenos resultados, incluso en pacientes con VEF1 $\leq 30 .{ }^{12}$

Tezacaftor es otro modulador que facilita el procesamiento y transporte intracelular de CFTR para aumentar la cantidad de proteína entregada a la superficie de la célula, lo que facilita la acción de ivacaftor y que, además, parece tener un perfil de seguridad mejorado en comparación con lumacaftor. En noviembre de 2017, se publicaron dos estudios fase 3 que demostraron la eficacia y seguridad de la combinación tezacaftor + ivacaftor $(\mathrm{T}+\mathrm{I}))^{13,14}$

El estudio EVOLVE se realizó con 510 pacientes homocigotos para la mutación F508del. El cambio absoluto y relativo en el porcentaje del VEF1 a favor de T + I sobre el placebo fue de 4 y 6,8 puntos porcentuales, respectivamente, y la tasa de exacerbación pulmonar fue un $35 \%$ menor en el grupo $\mathrm{T}+\mathrm{I}$ comparada con el grupo placebo $(\mathrm{p}=0,005){ }^{13}$

En el estudio EXPAND, con 248 pacientes mayores de 12 años, se incluyeron mutaciones heterocigotas para F508del y una mutación CFTR asociada a la función residual del canal. Se analizaron las intervenciones en grupos de pacientes, 162 para tezacaftor-ivacaftor, 157 para ivacaftor solo y 162 para placebo. El cambio del VEF1 (desde el valor basal hasta el promedio de la semana 4 y la semana 8 ) fue de 6,8 puntos porcentuales para $\mathrm{T}+\mathrm{I}$ y de 4,7 puntos porcentuales para ivacaftor solo $(\mathrm{p}<0,001$ para ambas comparaciones). Los puntajes en el CFQ-R también favorecieron significativamente a los grupos de tratamiento activo. La incidencia de eventos adversos fue similar en todos los grupos de intervención. ${ }^{14}$ La combinación tezacaftor + ivacaftor fue aprobada por la FDA en febrero de 2018 para su uso en pacientes con mutación homocigota F508del o una sola copia de 26 mutaciones específicas con actividad residual de la proteína. ${ }^{15}$

\section{EL DESAFÍO DE CREAR MODULADORES cystic fibrosis transmembrane conductance regulator}

Hasta la fecha, existen tres moduladores CFTR aprobados para su uso en niños (ivacaftor, ivacaftor + lumacaftor y tezacaftor + ivacaftor) y otros 11 que se encuentran aún en desarrollo, pero ¿cuál es la razón por la que no hay una mayor disponibilidad de moduladores CFTR? La respuesta se centra en la dificultad que, ciertamente, significa llevar a cabo cada una de las fases de estudio, desde la fase preclínica hasta los estudios de fase tardía.

\section{Fase preclínica, la primera tarea}

La implementación terapéutica de moduladores CFTR requiere del estudio genético del defecto de la proteína, el cual, muchas veces, no se encuentra al alcance de todos los pacientes o incluso para los sistemas de salud. ${ }^{16}$

- Cuando se va a probar un nuevo modulador, cuya mutación objetivo ya ha sido testeada para otro fármaco, se enfrenta la dificultad de comparaciones placebo-controladas a largo plazo. ${ }^{16}$

- Los moduladores deben ser sometidos a una evaluación preclínica que compruebe que su actividad observada se asocia con una modulación de CFTR que, probablemente, sea igual o supere las terapias aprobadas en la actualidad para contar con el apoyo económico o crear inquietud por parte de la comunidad de pacientes, que, para el caso de FQ, ha movilizado un gran interés sobre la investigación de nuevas terapias. ${ }^{16}$

\section{Estudios de fase temprana, biomarcadores y seguridad}

- La evaluación de seguridad debe demostrar que todos los componentes y las cantidades utilizadas son indispensables para obtener la eficacia correspondiente al régimen y dosis sugeridas del modulador en estudio. ${ }^{16}$

- Los biomarcadores utilizados deben ser seleccionados de manera que permitan la menor muestra de sujetos posible y que puedan predecir un efecto clínicamente importante en estudios posteriores con una muestra más grande. Al respecto, en la actualidad, se utilizan 3 biomarcadores, la diferencia de potencial nasal, cloro en sudor y $\mathrm{pH}$ duodenal, de los cuales el cloro en sudor parece ser el más sensible y de mejor alcance para su realización. ${ }^{16}$

- La medición de cloro en sudor tiene atributos relevantes: está estandarizado, no es invasivo, tiene baja variabilidad (clave para su extrapolación a poblaciones de mayor número) y es muy sensible. A pesar de no ser predictivo de valores VEF1 en individuos, 
sí existe evidencia fuerte que lo relaciona con cambios substanciales de VEF1 a nivel poblacional. ${ }^{16}$

- Un estudio publicado en 2017 propuso que la restauración funcional del canal CFTR, medida en cultivos de epitelio nasal humano podía predecir beneficios clínicos a largo plazo. El estudio utilizó los biomarcadores de diferencia de potencial nasal y expresión de CFTR (medida por fluorescencia) en cultivos de epitelio nasal humano en lugar de cultivos de epitelio bronquial. El resultado demostró que el nivel de corrección de CFTR se correlacionó significativamente con el cambio del VEF1 a los 6 meses en 8 pacientes tratados con moduladores de CFTR. ${ }^{17}$

\section{Estudios de fase tardía, demostración de eficacia clínica}

- Los investigadores deben desarrollar los estudios ajustándose a la ética, sobre todo, en los casos en los cuales ya existe una terapia aprobada como standard of care, y la discusión se centra en si se deben llevar a cabo estudios placebo-controlados. Esto se debe a que los estudios de comparación activa pueden realizarse solo después de determinar que no hay una población sin manejo previo (naíf), para la cual se podría llevar a cabo un ensayo controlado con placebo (por ejemplo: las poblaciones internacionales para las que el fármaco no está aprobado o disponible). Adicionalmente, pueden desarrollarse cuando los pacientes o los médicos tratantes no se oponen a que un caso dado pueda ser retirado del tratamiento de referencia actual y asignado a placebo durante un período determinado. ${ }^{16}$

- Cuando la decisión se incline a un estudio de comparación activa, existen tres nuevos desafíos que enfrentar: I) Se requiere un tamaño de muestra más grande en comparación a los ensayos placebo-controlado; II) Los posibles costos en los que se incurre para la adquisición y el cegamiento del comparador activo (blinding); III) Un grupo de comparación que es menos claro para la interpretación de los datos de seguridad. ${ }^{16}$

- El objetivo principal de estos estudios será un cambio significativo de las pruebas de función pulmonar, lo que bien podría considerarse un reto importante. ${ }^{16}$

\section{¿CUÁL ES EL FUTURO DE LOS MODULADORES cystic fibrosis transmembrane conductance regulator?}

El estado actual de las investigaciones de moduladores CFTR se encuentra ampliamente descrito en la página de la Cystic Fibrosis Foundation, en donde aparecen, además de los tres moduladores ivacaftor, lumacaftor + ivacaftor y tezacaftor + ivacaftor, otros 11 en diferentes fases de estudio.

- Dos en fase 3:

Tezacaftor $(V X-661)+$ ivacaftor + VX445

Tezacaftor (VX-661) + ivacaftor + VX659

- Cinco más en fase 2: QBW251, FDL169, GLPG2222, PTI428 (amplificador), vx561.

- Y 4 últimos en fase 1: PTI801, PTI808, QR010, MRT2005.

\section{CONCLUSIÓN}

Existe un gran compromiso por parte de la comunidad científica en el mundo para el estudio de nuevos tratamientos en pacientes con FQ. La terapia con moduladores CFTR es una realidad en desarrollo que tiene como objetivo la medicina personalizada, que atiende muy específicamente la genética de cada individuo con $F Q$ que es candidato a tratamiento. Hasta el momento, se han encontrado medicamentos que representan un desafío en la práctica clínica, con la esperanza de tener mejores resultados en un futuro.

\section{Agradecimientos}

Departamento de Pediatría, Universidad Nacional de Colombia, Servicio de Neumología Pediátrica del Hospital de la Misericordia.

\section{REFERENCIAS}

1. Zemanick E, Ong T, Daines C, Dellon E, et al. Highlights from the 2015 North American Cystic Fibrosis Conference. Pediatr Pulmonol. 2016;51(6):650-7.

2. Derichs N. Targeting a genetic defect: cystic fibrosis transmembrane conductance regulator modulators in cystic fibrosis. Eur Respir Rev. 2013;22(127):58-65.

3. Solomon S, Marshall S, Ramsey B, Rowe S. Breakthrough Therapies: Cystic Fibrosis (CF) Potentiators and Correctors. Pediatr Pulmonol. 2015;50(Suppl 40):S3-13.

4. Barry P, Ronan N, Plant B. Cystic Fibrosis Transmembrane Conductance Regulator Modulators: The End of the Beginning. Semin Respir Crit Care Med. 2015;36(2):287-98.

5. Aslam AA, Higgins C, Sinha IP, Southern KW. Ataluren and similar compounds (specific therapies for premature termination codon class I mutations) for cystic fibrosis. Cochrane Database Syst Rev. 2017;1:CD012040.

6. Cystic Fibrosis Foundation. Drug Company Ends Ataluren Program for CF Nonsense Mutations (Bethesda: March 3, 2017) [Internet]. [Consulta: 4 de junio de 2017]. Disponible en: www.cff.org/News / News-Archive/2017/DrugCompany-Ends-Ataluren-Program-for-CF-NonsenseMutations/. 
7. Van Goor F, Hadida S, Grootenhuis PD, Burton B, et al. Rescue of $\mathrm{CF}$ airway epithelial cell function in vitro by a CFTR potentiator, VX-770. Proc Natl Acad Sci U S A. 2009;106(44):18825-30.

8. Cystic Fibrosis Foundation. FDA Approves Ivacaftor for 23 Additional CFTR Mutations (Bethesda: May 17, 2017) [Internet]. [Consulta: 5 de junio de 2017]. Disponible en: www.cff.org/ News / News-Archive / 2017/ FDAApproves-Ivacaftor-for-23-Additional-CFTR-Mutations/.

9. Clancy JP, Rowe SM, Accurso FJ, Aitken ML, et al. Results of a phase IIa study of VX-809, an investigational CFTR corrector compound, in subjects with cystic fibrosis homozygous for the F508del- CFTR mutation. Thorax. 2012;67(1):12-8

10. BoyleMP, BellSC, Konstan MW, McColleySA, etal. ACFTR corrector (lumacaftor) and a CFTR potentiator (ivacaftor) for treatment of patients with cystic fibrosis who have a phe508delCFTR mutation: a phase 2 randomised controlled trial. Lancet Respir Med. 2014;2(7):527-38.

11. Wainwright CE, Elborn JS, Ramsey BW, Marigowda $\mathrm{G}$, et al. Lumacaftor-ivacaftor in patients with cystic fibrosis homozygous for Phe508del CFTR. N Engl J Med. 2015;373(3):220-31.

12. Hubert D, Chiron R, Camara B, Grenet D, et al. Real- life initiation of lumacaftor/ivacaftor combination in adults with cystic fibrosis homozygous for the Phe508del CFTR mutation and severe lung disease. J Cyst Fibros. 2017;16(3):388-91.

13. Taylor-Cousar JL, Munck A, McKone EF, van der Ent CK, et al. Tezacaftor-Ivacaftor in Patients with Cystic Fibrosis Homozygous for Phe508del. $N$ Engl J Med. 2017;377(21):2013-23.

14. Rowe SM, Daines C, Ringshausen FC, Kerem E, et al. Tezacaftor-Ivacaftor in Residual-Function Heterozygotes with Cystic Fibrosis. N Engl J Med. 2017;377(21):2024-35.

15. Cystic Fibrosis Foundation. FDA Approves New CFTR Modulator Treatment for Cystic Fibrosis (Bethesda: Feb 12, 2018) [Internet]. [Consulta: 12 de julio de 2018]. Disponible en: https: / / www.cff.org/News / News-Archive/2018/ FDA-Approves-New-CFTR-Modulator-Treatment-forCystic-Fibrosis/.

16. Mayer-Hamblett N, Boyle M, VanDevanter D. Advancing clinical development pathways for new CFTR modulators in cystic fibrosis. Thorax. 2016;71(5):454-61.

17. Pranke IM, Hatton A, Simonin J, Jais JP, et al. Correction of CFTR function in nasal epithelial cells from cystic fibrosis patients predicts improvement of respiratory function by CFTR modulators. Sci Rep. 2017;7(1):7375. 